\title{
Study of the influence of Carbonyl iron particulate size as an electromagnetic radiation absorbing material in 12.4 to $18 \mathrm{GHz}\left(\mathrm{K}_{\mathrm{u}}\right)$ Band
}

\author{
Ana Paula S. Oliveira ${ }^{1}$, Aline C. Rodrigues ${ }^{1}$, Sandro F. Quirino ${ }^{2}$, Diego Edissón F. Vergara ${ }^{1}$, Simone $^{3}$ \\ S. Pinto ${ }^{3}$, Mirabel C. Rezende ${ }^{3}$, Mauricio R. Baldan ${ }^{1}$ \\ ${ }^{I}$ Nacional Institute of Space Research, São José dos Campos, São Paulo, Brazil, \\ silvadeoliveira.ana@gmail.com, alinerodrigues_1@msn.com,diegoeflz@gmail.com, and \\ mrbaldan@gmail.com, \\ ${ }^{2}$ ETEP Faculty, São José dos Campos, São Paulo, Brazil, sandro10quirino@gmail.com, \\ ${ }^{3}$ Federal University of São Paulo, São José dos Campos, São Paulo, Brazil, simonesouza.pn@gmail.com and \\ mirabelcr@gmail.com
}

\begin{abstract}
This paper presents the influence of different sizes of carbonyl iron particles on the reflectivity measurements of Radar Absorbing Material (RAM). The electromagnetic characterization was performed with a vector network analyzer and a rectangular waveguide in the frequency range of 12.4 to $18 \mathrm{GHz}$ (Ku Band). The influence of different parameters such as thicknesses, particle sizes and concentration of carbonyl iron were evaluated. Reflectivity results showed the influence of these parameters on the performance of the RAM. The best reflectivity values $(\sim-18 \mathrm{~dB})$ were obtained for samples with 60 wt\% concentration and $5 \mathrm{~mm}$ thickness. We provide information about significantly reflection loss improvement by simply controlling carbonyl iron particulate size.
\end{abstract}

Index Terms - carbonyl iron filler, Ku band, microwave absorber.

\section{INTRODUCTION}

Radar Absorbing Material (RAM) is a type of material designed to attenuate electromagnetic radiation on specific frequencies. Many researches have been made about RAM due its countless applications in electromagnetic compatibility and interference reduction. These materials can be applied on communication systems, modern electronic devices, anechoic chamber, military stealth technology, and so on [1]. RAMs can be produced in different forms, such as paints or thin films [2]. Usually, RAMs are composite materials made with polymer (matrix) and absorptive material (mean). In the literature, materials with dielectric and/or magnetic losses are commonly used as means. In this matter, materials like ferrite, carbonyl iron (CI), carbonaceous materials and conductive polymers has advantages over lossless materials [2],[3]. Despite their high specific mass, composites made with ferrites or CI have advantages like thin thickness and broadband frequency absorption because of iron on their compositions [4]. Carbonyl iron has a relatively low electrical conductivity, a high Curie temperature, and a high saturation magnetization. These properties make CI a good candidate to be used as absorption mean, especially in the frequency range between $2-18 \mathrm{GHz}$ [5].

The effectiveness of the absorptive material contributes to the reflectivity losses that are determined 
through the obtained values of the complex permittivity and permeability. However, the control of the complex permittivity and permeability are obtained by the addition of magnetic and dielectric additives. Understanding the effects caused by RAM processing require the investigation of several parameters, but the main ones are absorbent mean and matrix properties [6]. Thus, this paper presents the influence of CI particle size on RAM absorption parameter over the frequency range from 2 to $18 \mathrm{GHz}$. Here, we propose the reflectivity control using different CI particle sizes, where we can significantly improve the absorption properties.

\section{EXPERIMENTAL}

\section{A. Material and sample preparation}

Composites were prepared with commercial CI powder from BASF GmbH as additive. Commercial bi-component silicone was used as matrix.

Carbonyl iron powder was separated into different particle sizes (Pe) using sieve shaker with three different sieves: $25<\mathrm{Pe}<53 \mu \mathrm{m} ; 53 \leq \mathrm{Pe}<63 \mu \mathrm{m}$ and; Pe $>63$. After 30 minutes sieving, CI powders were separated following the mass ratio of 40:60, 50:50 and 60:40 of CI concentration over silicone. Sample thickness ranged from $1 \mathrm{~mm}$ to $5 \mathrm{~mm}$, but since the best results were obtained for 2, 3 and $5 \mathrm{~mm}$, our discussions were based on these thicknesses. Table 1 summarizes all samples discussed in this paper.

TABLE I. SIZE OF THE PARTICULATES

\begin{tabular}{ccccccc}
\hline $\begin{array}{c}\text { Thickness of } \\
\text { samples (mm) }\end{array}$ & \multicolumn{3}{c}{ Size of CI particles Pe $(\boldsymbol{\mu m})$} & \multicolumn{3}{c}{ CI Concentration } \\
2 & $\mathrm{Pe}>63$ & $53<\mathrm{Pe}<63$ & $25<\mathrm{Pe}<53$ & $40 \%$ & $50 \%$ & $60 \%$ \\
3 & $\mathrm{Pe}>63$ & $53<\mathrm{Pe}<63$ & $25<\mathrm{Pe}<53$ & $40 \%$ & $50 \%$ & $60 \%$ \\
5 & $\mathrm{Pe}>63$ & $53<\mathrm{Pe}<63$ & $25<\mathrm{Pe}<53$ & $40 \%$ & $50 \%$ & $60 \%$ \\
\hline \hline
\end{tabular}

Composites were manually homogenized. Each sample of silicone-CI composite was mixed with $\sim 0.6 \mathrm{ml}$ catalyst until the beginning of reaction, which could be noticed by bubble formation and viscosity increasing. Before sample hardening, mixture was moved to $\mathrm{K}_{\mathrm{u}}$-band mold with $15.7 \mathrm{~mm}$ width and $7.9 \mathrm{~mm}$ height. Full catalyst reaction of composite mixtures was about 30 minutes.

\section{B. Morphological and electromagnetic characterization}

Crystalline phases of samples were investigated through X-Ray Diffraction (XRD). A Panalytical X'Pert Powder system equipped with a $\mathrm{CuK} \alpha(\lambda=0.154 \mathrm{~nm})$ was used. Composites were scanned from $20^{\circ}$ to $90^{\circ}$ with sampling intervals of $0.02^{\circ}$. Carbonyl iron particulate sizes were analyzed with a Field Emission Gun Scanning Electron Microscope (FEG-SEM). The equipment used was a TESCAN 
- Vega 3 operating with secondary electron detection.

Electromagnetic characterization was performed with a $\mathrm{K}_{\mathrm{u}}$-band rectangular waveguide (Agilent WR-62 P11644A) coupled on a $50 \mathrm{GHz}$ PNA-L vector network analyzer (Keysight N5232A). Electromagnetic properties were measured in the $\mathrm{K}_{\mathrm{u}}$ band, i.e., from 12.4 to $18 \mathrm{GHz}$. Through scattering parameters (S parameters) it was possible to comprehend the interaction of electromagnetic wave on samples and calculate the permittivity and permeability of materials. The method used to obtain the electromagnetic properties of samples was Nicolson Ross Weir (NRW), which is also called Transmission and Reflection (TR) method [7]. Fig. 1 illustrates the transmitted (S21 and S12) and reflected (S11 and S22) waves that can be measured with PNA-L. It is through these parameters that permittivity and permeability are extracted, allowing the material characterization over a frequency range.

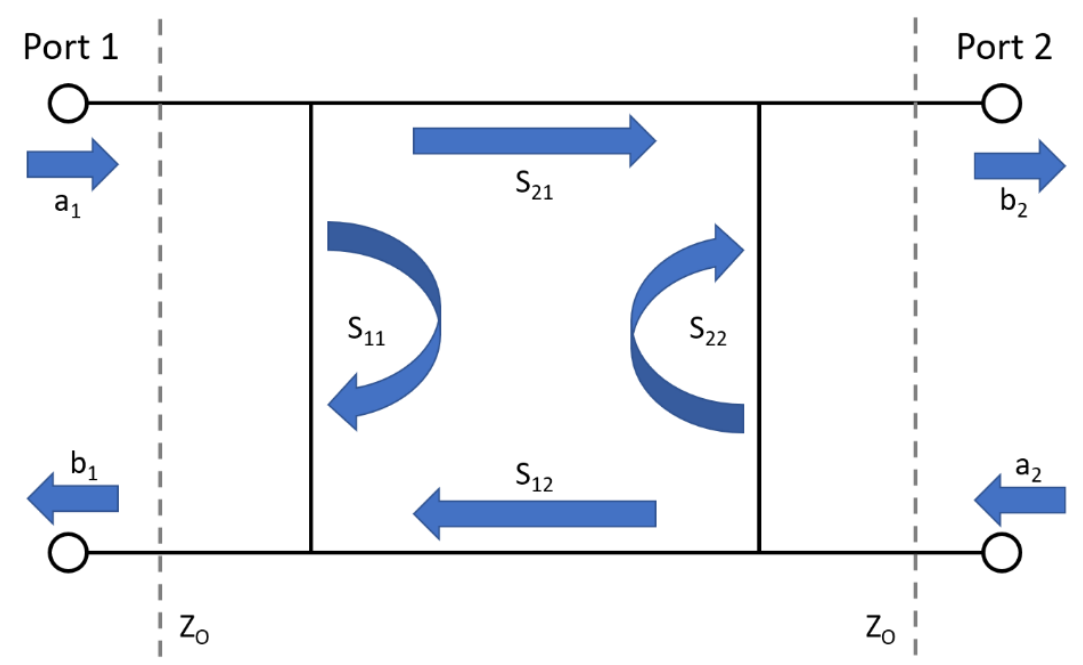

Fig.1 Schematic representation of a device with two ports [8]. The symbols a and b represent the amplitude of the incident wave and the response.

The electromagnetic properties of a material, i.e., the complex relative permittivity $\left(\boldsymbol{\varepsilon} \mathbf{r}=\boldsymbol{\varepsilon}{ }^{\prime}-\mathbf{j} \boldsymbol{\varepsilon}^{\prime \prime}\right)$ and permeability $\left(\boldsymbol{\mu} \mathbf{r}=\boldsymbol{\mu}^{\prime}-\mathbf{j} \boldsymbol{\mu}^{\prime \prime}\right)$ are determined by S-parameters measured over a frequency range [9]. The real part of permittivity and permeability ( $\boldsymbol{\varepsilon}^{\prime}$ and $\left.\boldsymbol{\mu}^{\prime}\right)$ represents the capacity of energy storage in the sample, while the imaginary parts $\left(\boldsymbol{\varepsilon}^{\prime \prime}\right.$ and $\left.\boldsymbol{\mu}^{\prime \prime}\right)$ represent the electric and magnetic energy losses [10].

\section{RESULTS AND DISCUSSIONS}

\section{A. Field Emission Gun Scanning Electron Microscopy}

Particulate sizes of pure carbonyl iron analyzed with FEG-SEM are presented in Fig. 2. All particulate sizes obtained after sifting presents spheres of different sizes. Although spheres apparently have similar sizes, there are different agglomerations of them over sifted powder. It is interesting to note that particulate sizes between $25 \mu \mathrm{m}$ and $53 \mu \mathrm{m}$, Fig. 2a, presents grains more dispersed than 
particulates sizes bigger than $63 \mu \mathrm{m}$. This agglomeration caused the particle separation through the sieves, where the average size of spheres clusters was estimated based on the sieve weft spacing.
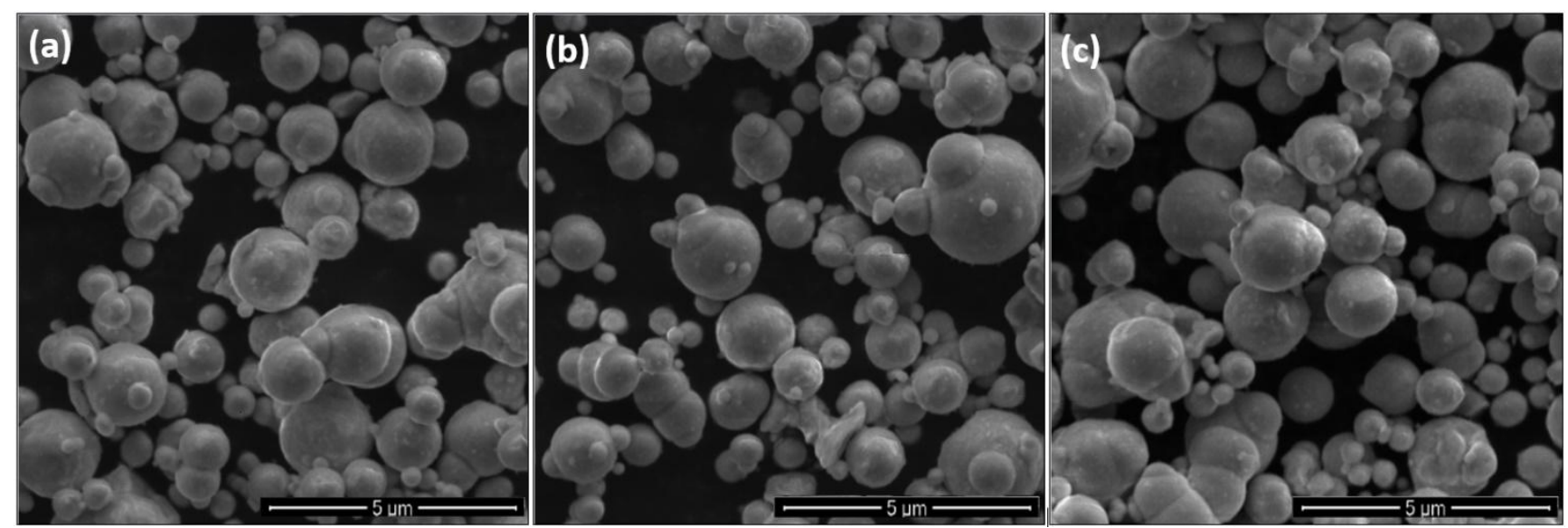

Fig. 2 FEG-SEM images obtained for the three particulate sizes with 15.000 of magnifications of (a) $25<\mathrm{Pe}<53$, (b) $53<$ $\mathrm{Pe}<63$, and (c) $\mathrm{Pe}>63 \mu \mathrm{m}$.

\section{B. X-Ray Diffraction}

XRD analysis of CI confirmed the same cubic crystalline lattice for all three separated particulate powder. The same result was obtained with CI powder with no sifting treatment, which means that crystalline structure was maintained after sifting and there was not a specific element responsible for agglomeration. Well defined peaks associated with (110), (200) and (211) planes were detected at $2 \theta$ $=44.6^{\circ}, 64.9^{\circ}$ and $82.3^{\circ}$, respectively. All peaks are related to the $\alpha$-Fe phase of the CI [9][10].

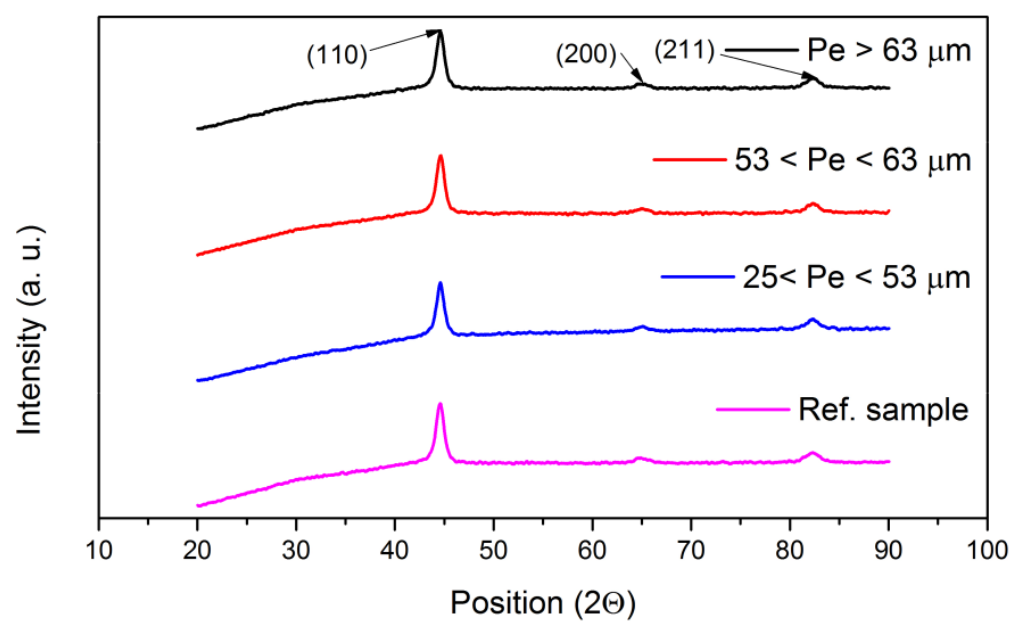

Fig.3 X-ray diffractograms of CI samples as a function of particle size (a) $25<\mathrm{Pe}<53 \mu \mathrm{m}$ (b) $53<\mathrm{Pe}<63 \mu \mathrm{m}$ (c) $\mathrm{Pe}>63$ $\mu \mathrm{m}(\mathrm{d})$ without sieving

\section{Electromagnetic Characterization - Electrical Permittivity and Magnetic Permeability}

Fig. $4 \mathrm{a}-4 \mathrm{c}$ represent the real permittivity $\left(\boldsymbol{\varepsilon}^{\prime}\right)$ of composites with CI concentrations of $40 \%, 50 \%$ Brazilian Microwave and Optoelectronics Society-SBMO Brazilian Society of Electromagnetism-SBMag received 30 Sept 2018; for review 14 Nov 2018; accepted 20 Nov 2018 $\begin{array}{lll}\text { (C) } 2018 \text { SBMO/SBMag } & \text { (cc) BY } \\ \end{array}$ 
and $60 \%$, respectively. Fig. $4 d-4 f$ are the real permeability $\left(\boldsymbol{\mu}^{\prime}\right)$ of composites with CI concentrations of $40 \%, 50 \%$ and $60 \%$, respectively. It is possible to observe the effects of CI concentration and particle size on permittivity and permeability analysis, where some measurements also presented a frequency dependency over the analyzed range. It is important to notice that CI concentration has a strong dependence on permittivity, as well as on the boundary conditions between additive and interface. Increasing the CI concentration affects the real permittivity due the interaction between CI particles. The internal structure arrangement and the volume fraction of $\mathrm{CI}$ in the silicone is directly related to the interaction of the electromagnet wave into the composite, having influence on the storage charging in the presence of an applied electric field [11]. It is possible to observe that at a $40 \%$ $\mathrm{CI}$ concentration causes a variation on permittivity from 3.85 to 4.6 , Fig. 4 a. For concentration of $60 \%$ this variation on permittivity is about 4.75 and 5.35, Fig. 4c. However, for 50\% CI concentration, Fig. $4 \mathrm{~b}$, the permittivity ranges from 3.2 to 5.1 , which is the biggest permittivity variation observed. The highest values of $\boldsymbol{\varepsilon}^{\prime}$ are associated with the highest proportion of CI inside silicone matrix, which increases the number of $\mathrm{CI} /$ silicone interfaces. Permeability of samples with $40 \%$ and $60 \% \mathrm{CI}$ concentration have a slightly decreasing as a function of frequency for all particle sizes and thicknesses. However, carbonyl iron concentration of $50 \%$ has no frequency dependence like the others. Similar behavior was observed by Zhu et al over the frequency range of $2-18 \mathrm{GHz}$ [12]. 

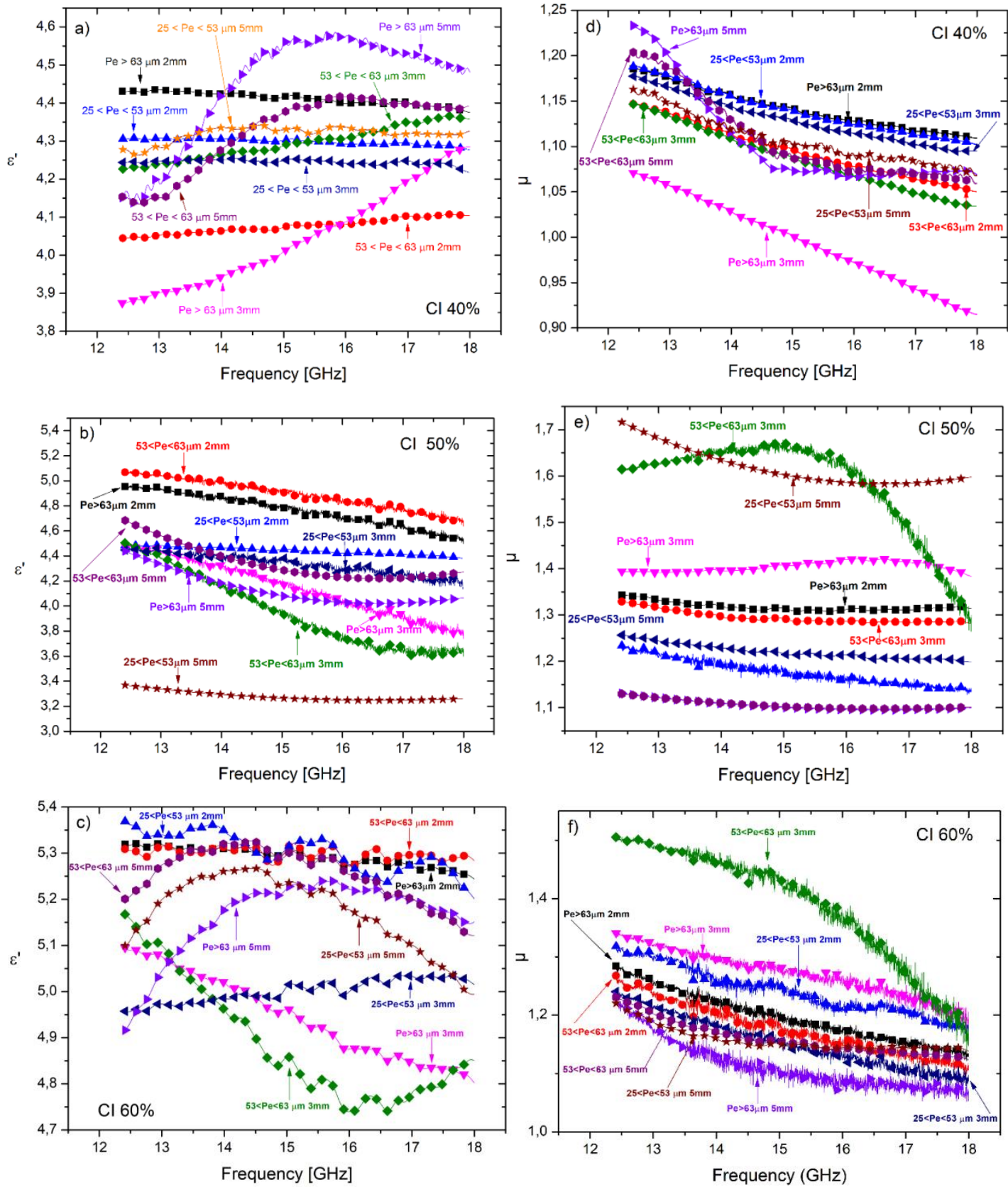

Fig.4 Experimental values of the real part of the permittivity (a) (b) and (c) and permeability (d), (e) and f as a function of frequency.

\section{Reflectivity Measures}

According to equations (1) and (2), the Reflection Loss (RL) of a RAM have influence of sample thickness and may have a frequency dependence inherited from complex permittivity and permeability [13].

$$
Z_{r}=\sqrt{\frac{\mu_{r}}{\varepsilon_{r}}} \tan \boldsymbol{h}\left(j \frac{2 \pi}{\lambda} t \sqrt{\mu_{r} \times \varepsilon_{r}}\right)
$$




$$
R L(d B)=20 \log \left|\frac{Z_{r}-1}{Z_{r}+1}\right|
$$

Here, $\boldsymbol{Z}_{\boldsymbol{r}}$ is the relative input impedance of material, $\mathbf{t}$ is the sample thickness, $\boldsymbol{\mu}_{\boldsymbol{r}}$ is the material relative magnetic permeability, $\boldsymbol{\varepsilon}_{\boldsymbol{r}}$ is the material relative dielectric permittivity and $\lambda$ is the wavelength of the incident wave in the free space.

Fig. $5 \mathrm{a}-5 \mathrm{~d}$ present the experimental reflectivity of evaluated samples. From Fig. 5a to Fig. 5c, reflectivity is highlighted for samples with 3 and $5 \mathrm{~mm}$ thickness, where all three particulate variations and CI concentrations are plotted. Since samples with $2 \mathrm{~mm}$ thickness presented the best RL results, all samples with this thickness is plotted in Fig. 5d for comparison.
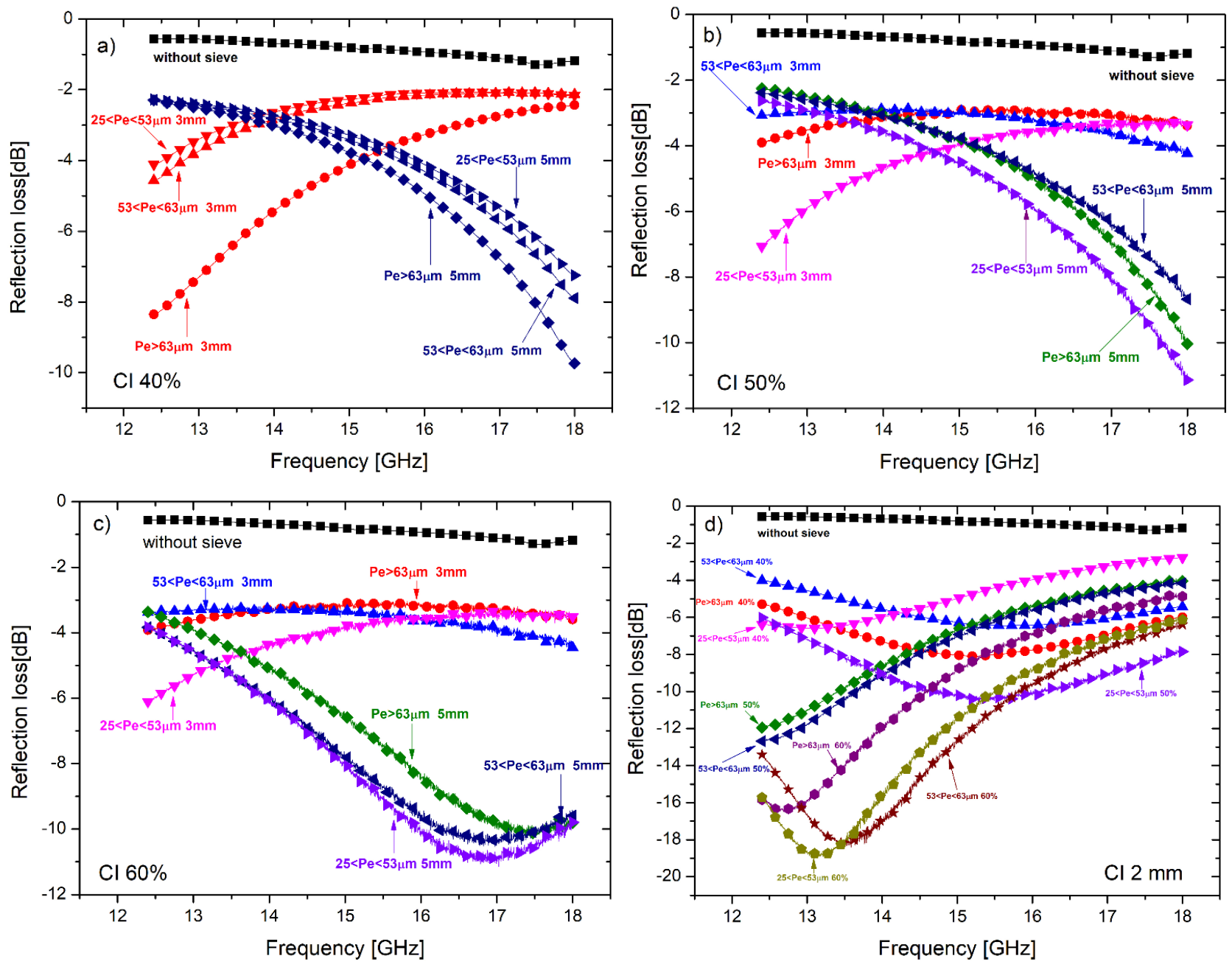

Fig. 5. (a), (b) and (c) reflectivity for samples of thicknesses of $3 \mathrm{~mm}$ and $5 \mathrm{~mm}$ with variation of the particulate, and (d) reflectivity for samples with variation of the particulate at $2 \mathrm{~mm}$ of thicknesses.

In Fig. 5a, which have RL plots of $40 \%$ concentration, it is possible to verify that the highest reflectivity value was obtained for sample with $5 \mathrm{~mm}$ thickness and particulate size bigger than 63 $\mu \mathrm{m}$. The attenuation in this sample is $-9.5 \mathrm{~dB}$ at $18 \mathrm{GHz}$, i.e., $90 \%$ of the incident wave was attenuated [14]. It is also observed that RAM with the same particle size, but with thickness of $3 \mathrm{~mm}$, tends to act as a microwave absorber in the X-band frequency range $(8.2-12.4 \mathrm{GHz})$. For these samples, the 
maximum reflectivity loss was $-8.5 \mathrm{~dB}$ at $12.4 \mathrm{GHz}$. Thus, RAM with $40 \% \mathrm{CI}$ concentration and $3 \mathrm{~mm}$ and $5 \mathrm{~mm}$ thicknesses tends to attenuate the incident wave in the X-band and K-band (18 $26.4 \mathrm{GHz})$, respectively.

This behavior is not observed for samples with no sieving. In other words, granulometric separation made possible the choice of the frequency band to work. This effect can be attributed exclusively to particle size, since no structural changes that could justify such behavior was noticed, as it could be observed in XRD graphs in Fig. 3.

It is possible to observe that $5 \mathrm{~mm}$ thickness samples trends to attenuate $\mathrm{K}$-band frequencies when samples present concentrations of $40 \%$ and 50\%, Fig. 5b. However, a minimum RL value is observed at $\sim 17 \mathrm{GHz}$ for CI concentration of $60 \%$, Fig. $5 \mathrm{c}$.

Samples with thickness of $2 \mathrm{~mm}$ have greater attenuations, which occurs mainly with CI concentration of $60 \%$. The best RL attenuation results are close to $13 \mathrm{GHz}$, Fig. $5 \mathrm{~d}$.

In summary, these results highlight different behaviors as a function of sample thickness and concentration. For the samples with $3 \mathrm{~mm}$ thickness, reflectivity presents a trend to have a better performance in the frequency band that precedes the $\mathrm{K}_{\mathrm{u}}$-band, i.e., X-band. For samples with $5 \mathrm{~mm}$ thickness there is a trend to great reflectivity attenuation in the K-band. These results show that the thickness and the concentration have a strong influence on frequency range division. These results are very important since controlling particulate size and thickness enable controlling of frequency ranges. For the same CI concentration (60\%) and two different thickness (2 and $5 \mathrm{~mm}$ ) it is possible to shift the RL from $13 \mathrm{GHz}$ to $17 \mathrm{GHz}$. The reflectivity curves show the predominant influence of the particle sizes resulting in more efficient absorbers with attenuation values $>90 \%$.

\section{CONCLUSIONS}

We demonstrated the possibility to attenuate electromagnetic wave over a frequency range by controlling sample thickness, particle size and CI concentration of RAM. Values obtained for reflectivity (RL) showed that it is possible to have an efficient RAM (RL $<-10 \mathrm{~dB})$ in the frequency range of $12-18 \mathrm{GHz}$ using a thin sample $(2 \mathrm{~mm})$ by simply controlling particulate size and concentration. Based on these results it is possible to design and manufacture different electromagnetic absorbers with the same material by simply controlling parameters of concentration particle size. Carbonyl iron separated in different particulates have great potential to be used as absorber materials in the $\mathrm{K}_{\mathrm{u}}$-band.

\section{ACKNOWLEDGMENT}

The authors would like to thank the Nacional Institute of Space Research and Materials Department (LABAS) for the support.

\section{REFERENCES}

[1] B. Wang, J. Wei, Y. Yang, T. Wang, and F. Li, "Investigation on peak frequency of the microwave absorption for carbonyl iron/epoxy resin composite," J. Magn. Magn. Mater., vol. 323, no. 8, pp. 1101-1103, 2011.

Brazilian Microwave and Optoelectronics Society-SBMO received 30 Sept 2018; for review 14 Nov 2018; accepted 20 Nov 2018 $\begin{array}{lllll}\text { Brazilian Society of Electromagnetism-SBMag } & \text { (c) } 2018 \text { SBMO/SBMag } & \text { (cc) BY } & \text { ISSN 2179-1074 }\end{array}$ 
[2] Y. Qing, W. Zhou, F. Luo, and D. Zhu, "Microwave-absorbing and mechanical properties of carbonyl-iron/epoxysilicone resin coatings," J. Magn. Magn. Mater., vol. 321, no. 1, pp. 25-28, 2009.

[3] D. Micheli, A. Vricella, R. Pastore, and M. Marchetti, "Synthesis and electromagnetic characterization of frequency selective radar absorbing materials using carbon nanopowders," Carbon N. Y., vol. 77, pp. 756-774, 2014.

[4] Y. Xu, D. Zhang, J. Cai, L. Yuan, and W. Zhang, "Effects of Multi-walled Carbon Nanotubes on the Electromagnetic Absorbing Characteristics of Composites Filled with Carbonyl Iron Particles," J. Mater. Sci. Technol., vol. 28, no. 1, pp. 34-40, 2012.

[5] C. C. Chen, W. F. Liang, Y. H. Nien, H. K. Liu, and R. Bin Yang, "Microwave absorbing properties of flakeshaped carbonyl iron/reduced graphene oxide/epoxy composites," Mater. Res. Bull., vol. 96, pp. 81-85, 2017.

[6] O. Khani, M. Z. Shoushtari, K. Ackland, and P. Stamenov, "The structural, magnetic and microwave properties of spherical and flake shaped carbonyl iron particles as thin multilayer microwave absorbers," J. Magn. Magn. Mater., vol. 428, no. December 2016, pp. 28-35, 2017.

[7] A. M. Nicolson and G. F. Ross, "Measurement of the Intrinsic Properties Of Materials by Time-Domain Techniques," IEEE Trans. Instrum. Meas., vol. 19, no. 4, pp. 377-382, 1970.

[8] M. A. do Amaral Junior et al., "Influence of the Permittivity on Carbon Fiber Particulates Applied in Radiation Absorbing Materials," Glob. J. Res. Eng. F Electr. Electron. Eng., vol. 17, no. 8, pp. 1-7, 2017.

[9] T. H. Min, H. J. Choi, N. H. Kim, K. Park, and C. Y. You, "Effects of surface treatment on magnetic carbonyl iron/polyaniline microspheres and their magnetorheological study," Colloids Surfaces A Physicochem. Eng. Asp., vol. 531, no. July, pp. 48-55, 2017.

[10] S. Tyagi, H. B. Baskey, R. C. Agarwala, V. Agarwala, and T. C. Shami, "Development of hard/soft ferrite nanocomposite for enhanced microwave absorption," Ceram. Int., vol. 37, no. 7, pp. 2631-2641, 2011.

[11] Y. Qing, D. Min, Y. Zhou, F. Luo, and W. Zhou, "Graphene nanosheet- and flake carbonyl iron particle-filled epoxy-silicone composites as thin-thickness and wide-bandwidth microwave absorber," Carbon N. Y., vol. 86, pp. 98-107, 2015.

[12] Y. C. Qing, W. C. Zhou, S. Jia, F. Luo, and D. M. Zhu, "Electromagnetic and microwave absorption properties of carbonyl iron and carbon fiber filled epoxy/silicone resin coatings," Appl. Phys. A Mater. Sci. Process., vol. 100, no. 4, pp. 1177-1181, 2010.

[13] E. F. Knott, J. F. Shaeffer, and M. T. Tuley, Radar Cross Section. 2004.

[14] S.M. Lee, "International encyclopedial of composites", v.6, New York: VHC Publishers, 1991. 